\title{
A Typical Analysis of Flying Ad-Hoc Network Applications in Agriculture and Irrigation System
}

\author{
Caprio Mistry $^{1 *}$, Ahona Ghosh ${ }^{2}$, Mousumi Biswas ${ }^{3}$, Hitender Vats ${ }^{4}$ \\ ${ }^{1}$ Department of Electronics and Communication Engineering, Brainware University, Kolkata, India \\ ${ }^{2}$ Department of Computational Science, Brainware University, Kolkata, India \\ ${ }^{3}$ Department of Computer Science and Engineering, Brainware University, Kolkata, India \\ ${ }^{4}$ Department of Electronics and Communication Engineering, ITM University Gwalior, India \\ ${ }^{1 *}$ research.caprio@gmail.com
}

\begin{abstract}
With the speedy technological enhancement and decrement in man power, technologies are being involved in every phase of our life and their proper application leads to a healthy life indeed. Agriculture and irrigation are sources of man's potential. Different sensors and electronics equipment are deployed to commercialize in market and maintaining the cost effectiveness in several domains. For the sake of cost effectiveness and the betterment of agricultural professionals, UAVs (unmanned aerial vehicles) can be deployed for surveillance, utilization of pesticides and insecticides and detection of bioprocessing errors. Single mode and multi-mode UAV system both are suitable for application of FANET. With the proper collaboration and coordination of the clusters of UAVs forming a network, linked with a ground infrastructure, GCS or satellite will exceed the competences of single UAV system. However, one of the vital design issues FANETs deal with is in selecting the accurate routing protocol which is prerequisite for the creation of FANET. In this paper we will discuss about the routing protocols of FANET in different platforms and different strategic manners. The open research challenges will be discussed and possible solutions will be attempted to be drawn from conclusion. The main contribution lies in the suggesting the most suitable routing protocol for each particular agriculture application based on the mobility model and requirement.
\end{abstract}

\section{Introduction}

Precision farming is a revolutionary approach used by farmers to maximize inputs like water and fertilizers, improving efficiency, quality and yields. This definition also includes the minimization of pests and diseases by targeting spatially. A pesticide level precise agriculture enables the farmer to deal correctly with each part of his field enabling him to tackle crop diversification. The reason behind farmers being more correct about planting, harvesting and fertilization is it contributes to higher farm production and profitability while upholding environmental standards. Agriculture is an industry involving vast volume of data and gets influenced by disease of plant and pest appearance. These data are also linked to environmental quantitative characteristics like ambient temperature, soil temperature and chemical composition, precipitation, solar radiation and crop quality. List like this one reporting, coupled with appropriate processing techniques, would bring new opportunities for rural advancement. The information and communication technology (ICT) and its development will promote more data-oriented agriculture. Different advances such as the Internet of Things (IoT) [32] and cloud computing are mixed in sequence [26].

Intelligent data analysis and successful dissemination of knowledge to agricultural stakeholders and smart farming [39] are required to introduce productive data collection. In turn, geospatial tools such as the Global Navigation and Global Positioning System in precision agriculture even the satellite network (GNSS) will play an important role as they can indicate any component's exact position. Wireless sensor network (WSN) [1] [2] and AdHoc Network [3] [44], especially FANET (Flying AdHoc Network) [20], a new form of Mobile Ad Hoc Network (MANET) [45] is one of the efficient platforms which we can apply for betterment of agriculture. The increase in the production of UAVs [33] is also a very promising approach for the collection of realtime details, as it is considered as one of the most ambitious precision-farming technologies [46] [47]. UAVs are able to enforce a series of steps like data collection, data preparation, data interpretation and data management. Their willingness to travel makes them much more desirable, since they can get in a shorter period, useful knowledge and ground evaluation can't. The biggest downside is that the UAV is complex model which requires the synchronization of numerous scientific logics with technologies for successful functionality.

\section{Related works}

UAV implementation in agriculture results in six specific applications: crop scouting, crop survey and mapping, crop protection, crop planning and management, chemicals, geofencing, etc. Agriculture itself is one of the highly demanded domains due to the huge population density in our 
country. Among three different routing protocol categories of FANET [4], Destination Sequenced Distance Vector (DSDV) [5], Optimized Link State Routing (OLSR) [6] come under the proactive category where all the network nodes share routing information with all the other nodes in a regular time interval which helps to create the path to ultimate destination in turn. AdHoc On-Demand Distance Vector (AODV) [7], Associativity Based Routing (ABR) [25] and Dynamic Source Routing (DSR) [10] come under reactive protocol since they create path only when it is required and are the most popular and effective ones when applied to agriculture as they consume minimum bandwidth in the system. As the nodes are very frequently movable which results in frequently changing network topology, efficient routing is the most challenging part of FANET [8] along with the other challenges like Quality of Service (QoS) [23] [24], Security, Reliability, Network scalability etc. [9]. A comprehensive study of the existing routing protocols has been presented in [11] where it is clearly shown that the existing ones obtain promising result, but still there is a scope for further research on more reliable and robust routing protocol which can harness the potential of UAV in different application areas. Self-organization of UAVs has been implemented in [12] being influenced from swarm of birds to deal with the distributed and dynamic nature of FANET.

Packet forwarding technique, routing, mobility and traffic density are the considered parameters to evaluate the performance of FANET and simulation study on network delay along with load and throughput has evaluated various routing protocols mentioned in [13]. It is possible to distinguish UAVs into two major divisions (i) fixed wing and (ii) multirotor. A specified wing has better aerodynamics, lower energy consumption leading to longer flights and faster speed, but requires considerable space to take off and land. A multi-rotor can accommodate heavier payloads, piloting is smoother, as both the take-off and landing are carried out vertically. UAVs consist of six main sub-modules working together to create a beneficial platform. i) The unmanned aerial vehicles airframe acts as the body of the UAV, which must be light enough to sustain low energy consumption and sufficiently strong to support the increased UAV payload for accepting fatal incidents and crashes when avionics have small space and no storage for the pilot. ii) UAV's heart is the flight machine. It is used to collect aerodynamic knowledge by means of a set of sensors (GPS, accelerometers, gyroscope, magnetometers, etc.) to direct the UAV ride according to the ride plan from the airframe-mounted control board [14]. iii) The payload consists of a collection of actuators and sensors which is responsible for either processing the data after acquiring or transmitting it to the base station for further analysis. iv) The sensors which are a part of the payload get controlled by the payload/mission controller. v) The base station situated on the ground manages and controls the payload and UAV. vi) The communication platform consisting of different communication channels including microwave, radio etc. helps to establish the communication pathway between the base station and the UAVs in the network. UAV can be effective where application of traditional aids is quite challenging like sugarcane, pigeon pea, wet paddy field etc. [15].

Navigation based UAV weighing less than $14 \mathrm{Kg}$ has been used to process high temporal and spatial resolution of agricultural field-based image data in [17]. Experimental result showed only a $1.5 \%$ difference between the image assessed herbicide affected region and the herbicide affected region assessed by ground survey. A helicopter introduced in [18] got able to fly over previously set waypoints under the AFCS control and accuracy of the hovering reached $\pm 1 \mathrm{~m}$. The ADC image sensor could be triggered by controller in terms of GPS locations, the attitude and position of capturing images were recorded at the same time for post processing. FANET architectures should be capable of handling network fragmentation [21] and high mobility, low density are the main challenges faced by FANET in 3D spaces having different altitudes. Existing works have focused on any one of these issues, none has considered all of these combinedly. As there is no physical guidance towards various flight schematics and control in a drone, different factors like degree of autonomy, size and weight of the drone, difference in energy source (conventional aircraft or battery driven or solar fuel-based energy) matter during designing of the same [28]. Flight range, payload capacity, and cost are the other essential parameters since UAVs not only monitors the field, but also protects plants and applies pesticides according to the requirement [29] [31].

\section{Protocols of routing used in agriculture}

Implementation of routing protocols for FANET is a challenging task as it has to deal with three dimensional movements due to the dynamic nature of FANET [22]. The routing techniques follow any or combination of any of the following techniques and the outline has been shown in Fig.1.

i) Store carry and forwarding method: Network with a high fragmentation consists of some nodes which sometimes fail to find out relay node for transmission and start carrying the packet until an appropriate relay node is found which results in network delay in forwarding the required packet due to undesired movement of the node.

ii) Greedy method: The number of hops is the main criteria in selecting the path towards destination in this technique. High dense networks apply this technique to use the closest node to relay the packets efficiently within a specified time. 
iii) Single path method: In this technique, between two communicating nodes, there exists only one path where the maintenance of routing table for each node is easier than the other methods, but it results in packet loss when the one and only possible path exhibits some error.

iv) Multipath method: This method overcomes the loophole of single path method by introducing multiple paths, in case of failure of one path alternatives start functioning to avoid packet loss in the network, but routine table maintenance is little bit complex in this as it has to manage the tables for all the possible paths existing.

v) Path exploration method: When the source network node does not contain any information about the path towards destination node, the path needs to be explored using Route Request (RREQ) where either the source nodes get broadcasted to its neighbors or it introduces flooding technique where each node upon receiving RREQ, copies it and transmits to its neighbours. Upon receiving the RREQ, the destination node unicasts the route reply as an answer to the source node. In this way, the route gets discovered and gets applied in routing onwards.

vi) Forecasting based method: The node's speed, direction and geographical position are the three main criterion in this method to select the next relay node to a particular network node which in turn reduces the network delay and packet delivery ratio.

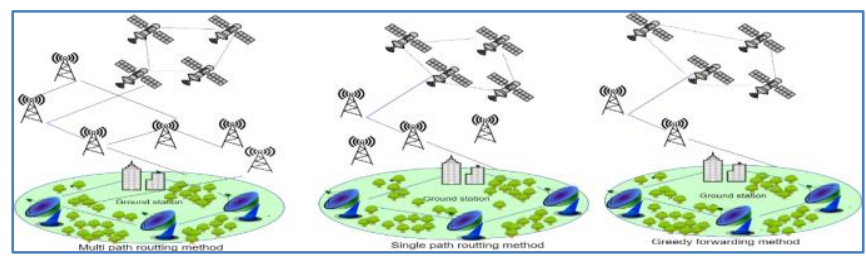

Fig.1. Mostly used routing architectures of FANET

Two categories of network architecture have been used in agricultural applications of FANET discussed in the existing literature.

i) Communication within air

Infrastructure less architecture needs this type of protocol where packet forwarding is required by UAV to an unreachable node. In a scenario where ground setup is not possible, its wide application can be observed.

ii) Communication between air and ground

When every UAV in a network is not able to communicate with an existing ground station due to their restriction in transmission range, relay nodes are applied to establish the connection within their transmission range.

The routing protocols are designed based on two parameters, the first one is its position where the location of each node is tracked by GPS which does not shown any additional advantage in highly mobile networks like FANET and the second one is the network topology which is extensively applied in the existing literature for remote sensing of irrigation system using FANET. The two categories of routing are described as follows

\subsection{Routing based on position}

AODV, DSR, TORA, M-OLSR, NC-OLSR, D-OLSR, CEOLSR, DSDV, OLSR have been widely adopted in FANET's application in agriculture. Coordination mechanism for different nodes within FANET is shown in Fig.2.

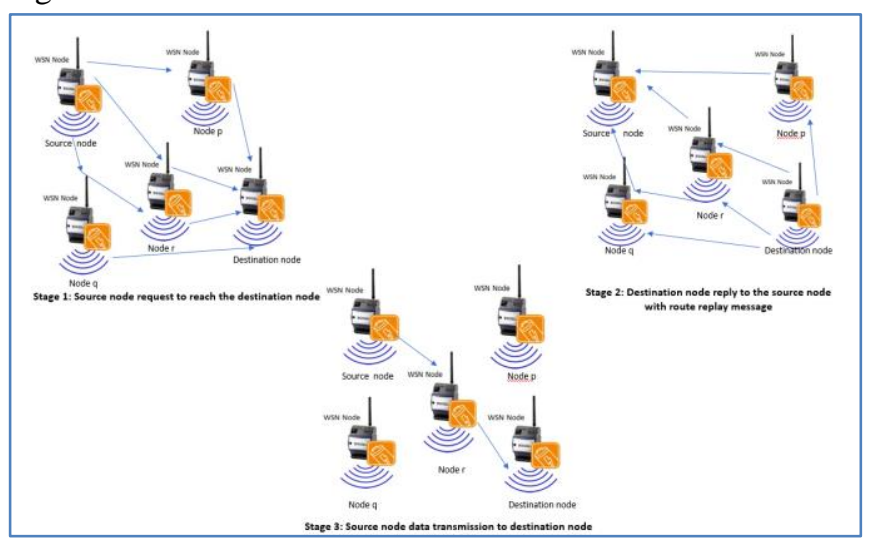

Fig. 2. Coordination mechanism between FANET nodes

\subsubsection{Robust and Reliable Predictive Routing (RARP)}

Growing requirement of compact and flexible communications have led to rapid growth in networking between unmanned aerial vehicles (UAVs) also referred to as flying ad-hoc networks (FANETs). Established handheld ad-hoc routing protocols are not appropriate for FANETs due to high-speed mobility, environmental conditions and land structures. A new stable and efficient predictive routing system with lateral and dynamic angle change transmission for FANETs. Several new aspects have been clarified in detail, such as estimation of the planned link time, route selection usefulness, directional transmission with a new upgrade method, dynamic angle change with the use of an adjustable antenna, alternate path configuration, and local path repair.

\subsubsection{Jamming Resilient Multipath Routing}

Jamming attacks are extremely dangerous to reliability. Wireless correspondence, as can be easily interrupted Contact. Communication. Current jamming defenses rely mainly on restoring communication between neighboring nodes. As long as all routes do not collapse at the same time, the availability of end-to-end paths is retained. Wireless networks interact through a common media and are thus susceptible to interference with jamming and radio. A great deal of research has been done to repair the jamming locally, i.e., to restore communication between neighboring nodes. 
These measures include common physical-layer methods based on specialized transceiver [35] (for example, frequency hopping) and MAC-layer mechanisms [36,37] modifying error coding, channel adaption [37] or physical location [38]. These measures are anti-jamming. Though we have to protect those strategies against jamming, different perspective to emphasize on defense Network level jamming, i.e., restoring end-to-end data transmission stability can be considered.

\subsubsection{Ground Control System based Routing}

As per Ad Hoc network communication the inherent shortcomings of MANET protocol that were not suitable for FANET were unavoidably inherited. Based on GCS advantages and suggested GCS routing specially designed for FANET in order to eliminate those limitations.

\subsubsection{Adaptive Beacon Scheme for Geographic Routing}

Based on mobility prediction, it consists of a fuzzy controller to adjust the beacon advertisement frequency adaptively where the error degree in prediction works as the input and beacon interval rate as the output. Its feats the time and location history of nearby FANETs applying weighted linear regression model [40]. To reach the target, the nearest or the next to nearest UAV is chosen as the next hop destination in each step

\subsection{Routing based on topology}

Topology aware conventional routing protocols can be categorized into three types, proactive, reactive and hybrid protocol. Continuously moving FANET systems require dynamic topology to avoid interruptions and delays in the network communication [41]. Most of the MANET protocols get applied in FANET architecture by integrating GPS positioning functionalities and to deal with complex situations, sometimes integration of more than one routing protocols takes place as per the user requirement. Following discussion is based on some recently applied routing protocols in FANET architecture [48].

\subsubsection{Distributed Priority Tree Based Routing Protocol}

The issues faced by aero-ground ad-hoc networks for network segmentation have been considered by Sharma et al. [34] to introduce a protocol modifying the basic r-b structure suitable for communication and routing two selforganizing nodes within a coordinate system. At first it identifies the ground nodes, then air nodes get detected by it and finally the interface between air and ground gets identified using a neural network algorithm which generates a routing table in the form of a tree containing air nodes and ground nodes.

\subsubsection{Continuous Hopfield Neural Network Optimization}

Probabilistic models have worked efficiently in handling and modeling different natural phenomena as close as possible to reality. While most of the other routing protocols work based on count of hops [42], continuous Hopfield/Feedback neural network tries to find out the shortest route visiting all the cities at least once based on the travelling salesman algorithm in the network. However, the applicability of such model has not been tested yet for greater number of nodes and the utility function can also be modified based on characterizing the level of the link's satisfaction in FANET [43].

\subsubsection{Topology Construction Method}

Topology construction method of routing has also proved its efficiency in reducing energy consumption for WSN in the existing literature.

\subsubsection{Topology Change Aware Routing Choosing Scheme}

Mobility measuring metric namely topology change degree helps to describe the topology changes in highly dynamic FANETs which is capable of optimizing average jitter, average end-to-end delay, network throughput, and packet delivery ratio also. However, in future some research challenges are still to be addressed about effective setting of the topology change perception interval, accurate calculation of the topology change threshold and configuration of the weight factors among the influencing parameters, dealing with the problems of changes in the number of nodes?

\subsection{Routing based on swarm}

Different swarm intelligence-based routing protocols have been applied in existing precision agricultural frameworks [16] [19] [27]. Ant colony optimization, Particle swarm optimization, Honey bee based AODV are some of them which self-organize and efficiently divide labor to be capable of optimizing the network life span, adaptability, fault tolerance, scalability, energy and latency as well.

\section{Application domains in agriculture}

The various application domains of FANET in agriculture are described as follows

\subsection{Crop monitoring}

Crop monitoring is required at every stage of a plant's growth, from sprouts to harvest [30]. Farmers especially commercial agriculture practitioners face a challenge to monitor their crops in efficient time and energy. After deploying autonomous quadcopter UAV, the farmer can decide to check them weekly or on monthly basis based on the need. It functions mainly to checkmate pest infestation on plants and helps the farmer to identify plants that are not 
growing perfectly. The field inspection from sky gives a better view and allows the farmer to judge the crop requiring uprooting or treatment in an improved way. The toxic and harmful pesticides to human beings and the environment can be applied to the crops by UAV also.

\subsection{Ranching animal husbandry}

Cattle rearing can get easy by introduction of ranching during inspecting animals by UAV in timely manner.

\section{Conclusion}

This study has attempted to draw an outline of the improvement in overall product quality due to significant applications of FANET in smart farming. Exploitation of land, energy and the other resources can be reduced to a great extent by deploying drone technology in irrigation system. Development of new algorithms for peer-to-peer network configuration is suggested for collision control and congestion avoidance in such platform. In the last decade almost hundred number of routing protocols for FANET have been introduced by researchers having their own characteristics, benefits and shortcomings. Since the mobility in the highly dynamic model plays a vital role, they have been compared using taxonomy. Finally, some less explored areas have been identified as open research challenges for future.

\section{References}

1. Yick, J., Mukherjee, B. and Ghosal, D., 2008. Wireless sensor network survey. Computer networks, 52(12), pp.2292-2330.

2. Ghosh, A., Ho, C.C. and Bestak, R., 2020. Secured Energy-Efficient Routing in Wireless Sensor Networks Using Machine Learning Algorithm: Fundamentals and Applications. Deep Learning Strategies for Security Enhancement in Wireless Sensor Networks, pp.23-41.

3. Shobana, M.; Karthik, S. A Performance Analysis and Comparison of various Routing Protocols in MANET. In Proceedings of the 2013 International Conference on Pattern Recognition, Informatics and Mobile Engineering (PRIME), Salem, India, 21-22 February 2013.

4. Sahingoz O. Networking models in flying ad-hoc networks (FANETs): Concepts and challenges. Journal of Intelligent and Robotic Systems. 2014;74. DOI: 10.1007/ s10846-013-9959-7.

5. He, G., 2002. Destination-sequenced distance vector (DSDV) protocol. Networking Laboratory, Helsinki University of Technology, pp.1-9.
6. Singh K, Verma AK. Applying OLSR routing in FANETs. In: 2014 IEEE International Conference on Advanced Communications, Control and Computing Technologies; Ramanathapuram: 2014. Pp. 1212-1215.

7. Singh, K. and Verma, A.K., 2015, March. Experimental analysis of AODV, DSDV and OLSR routing protocol for flying adhoc networks (FANETs). In 2015 IEEE International Conference on Electrical, Computer and Communication Technologies (ICECCT) (pp. 1-4). IEEE.

8. Kashyap, K.K. and Agrawal, A., 2018. Fanet: Survey on Design Challenges, Application Scenario and Communication Protocols. IJRAR-International Journal of Research and Analytical Reviews (IJRAR), 5(4), pp.10241032.

9. Singh K, Verma AK. Flying adhoc networks concept and challenges. In: Khosrow-Pour M DBA, editor. Advanced Methodologies and Technologies in Network Architecture, Mobile Computing, and Data Analytics.

10. Khan M.A, Safi A, Qureshi I.M., Khan I.U., Flying adhoc networks (FANETs): A review of communication architectures, and routing protocols. In: 2017 First International Conference on Latest trends in Electrical Engineering and Computing Technologies (INTELLECT); Karachi: 2017. pp. 1-9

11. Lakew, D.S., Sa'ad, U., Dao, N.N., Na, W. and Cho, S., 2020. Routing in Flying Ad Hoc Networks: A Comprehensive Survey. IEEE Communications Surveys \& Tutorials, 22(2), pp.1071-1120.

12. Khare, V.R.; Wang, F.Z.; Wu, S.; Deng, Y.; Thompson, C. Ad-hoc network of unmanned aerial vehicle swarms for search \& destroy tasks. In Proceedings of the $4^{\text {th }}$ International IEEE Conference Intelligent Systems, Varna, Bulgaria, 6-8 September 2008.

13. Khan, M.A., Khan, I.U., Safi, A. and Quershi, I.M., 2018. Dynamic routing in flying ad-hoc networks using topology-based routing protocols. Drones, 2(3), p.27.

14. Kakamoukas, G.A., Sarigiannidis, P.G. and Economides, A.A., 2020. FANETs in Agriculture-A routing protocol survey. Internet of Things, p.100183.

15. Sinha, J.P., Kushwaha, H.L., Kushwaha, D., Singh, N. and Purushottam, M., 2016. Prospect of Unmanned Aerial Vehicle (UAV) Technology for Agricultural Production Management. IIT Kharagpur: Agricultural and Food Engineering Department, IIT Kharagpur.

16. Maes, W.H. and Steppe, K., 2019. Perspectives for remote sensing with unmanned aerial vehicles in precision agriculture. Trends in plant science, 24(2), pp.152-164. 
17. Xiang, H. and Tian, L., 2011. Development of a low-cost agricultural remote sensing system based on an autonomous unmanned aerial vehicle (UAV). Biosystems engineering, 108(2), pp.174-190.

18. Xiang, H. and Tian, L., 2006. Development of autonomous unmanned helicopter based agricultural remote sensing system. In 2006 ASAE Annual Meeting (p. 1). American Society of Agricultural and Biological Engineers.

19. Primicerio, J., Di Gennaro, S.F., Fiorillo, E., Genesio, L., Lugato, E., Matese, A. and Vaccari, F.P., 2012. A flexible unmanned aerial vehicle for precision agriculture. Precision Agriculture, 13(4), pp.517-523.

20. Bujari, A., Calafate, C.T., Cano, J.C., Manzoni, P., Palazzi, C.E. and Ronzani, D., 2017. Flying ad-hoc network application scenarios and mobility models. International Journal of Distributed Sensor Networks, 13(10), p.1550147717738192.

21. Oubbati, O.S., Atiquzzaman, M., Lorenz, P., Tareque, M.H. and Hossain, M.S., 2019. Routing in flying ad hoc networks: Survey, constraints, and future challenge perspectives. IEEE Access, 7, pp.81057-81105.

22. Wen, S. and Huang, C., 2018. Delay-constrained routing based on stochastic model for flying ad hoc networks. Mobile Information Systems, 2018.

23. Roy, D.G., Ghosh, A., Mahato, B. and De, D., 2018, July. QoS-Aware Task Offloading Using Self-organized Distributed Cloudlet for Mobile Cloud Computing. In International Conference on Computational Intelligence, Communications, and Business Analytics (pp. 410-424). Springer, Singapore.

24. Roy, D.G., Mahato, B., Ghosh, A. and De, D., 2019. Service aware resource management into cloudlets for data offloading towards IoT. Microsystem Technologies, pp.1-15.

25. Toh, C.K., 1997. Associativity-based routing for ad hoc mobile networks. Wireless Personal Communications, 4(2), pp.103-139.

26. Mukherjee, D., Chakraborty, S., Sarkar, I., Ghosh, A. and Roy, S., A Detailed Study on Data Centre Energy Efficiency and Efficient Cooling Techniques, International Journal of Advanced Trends in Computer Science and Engineering (IJATCSE), 9(5), pp. 9221-9242.

27. Sharma, R. and Mishra, J., 2020. Review on Application of Drone Systems in Precision Agriculture. Journal of Advanced Research in Electronics Engineering and Technology, 7(2), pp.5-7.

28. Essien, V., Azeta, J., Bolu, C. A., and Okokpujie, I.P., Application of Drones for Agricultural Processes: its
Challenges and Way-Forward, International Journal of Emerging Trends in Engineering Research (IJETER),8(10), pp. 7712-7717.

29. Marinello, F., Pezzuolo, A., Chiumenti, A. and Sartori, L., 2016. Technical analysis of unmanned aerial vehicles (drones) for agricultural applications. Engineering for Rural Development, 15.

30. Psirofonia, P., Samaritakis, V., Eliopoulos, P. and Potamitis, I., 2017. Use of unmanned aerial vehicles for agricultural applications with emphasis on crop protection: Three novel case-studies. International Journal of Agricultural Science and Technology, 5(1), pp.30-39.

31. Salamí, E., Barrado, C. and Pastor, E., 2014. UAV flight experiments applied to the remote sensing of vegetated areas. Remote Sensing, 6(11), pp.11051-11081.

32. Sowjanya, M., Decision Supporting System in Agriculture through IOT, International Journal of Emerging Trends in Engineering Research (IJETER),8(10), pp. 67386741.

33. Assylbekov, U., Baiseitov, G., Boiko, A., Temirbekuly, A., Orazkanov, A., The control system of a promising domestic unmanned aerial vehicle of the tactical management level, International Journal of Emerging Trends in Engineering Research (IJETER),8(10), pp. 69346939.

34. Sharma, V., Kumar, R. and Kumar, N., 2018. DPTR: Distributed priority tree-based routing protocol for FANETs. Computer Communications, 122, pp.129-151.

35. J. Proakis. Digital Communications. McGraw-Hill, Columbus, OH, 2000.

36. G. Noubir and G. Lin. Low-power DoS attacks in data wireless lans and countermeasures. SIGMOBILE Mob. Comput.

37. W. Xu, W. Trappe, and Y. Zhang. Channel surfing: defending wireless sensor networks from interference. In Proceedings of conference on Information Processing in Sensor Networks (IPSN), pages 499-508, 2007.

38. K. Ma, Y. Zhang, and W. Trappe. Mobile network management and robust spatial retreats via network dynamics. In Proceedings of workshop on Resource Provisioning and Management in Sensor Networks (RPMSN05), 2005.

39. Zainal, N., Mohamood, N., Norman, M.F. and Sanmutham, D., 2019. Design and implementation of smart farming system for fig using connectedargonomics. International Journal of Electrical \& Computer Engineering (2088-8708), 9(6). 
40. Albu-Salih, A.T. and Khudhair, H.A., 2021. ASRFANET : An adaptive SDN-based routing framework for FANET. International Journal of Electrical \& Computer Engineering (2088-8708), 11(5).

41. Sadiq, B.O. and Salawudeen, A.T., 2020. FANET optimization: a destination path flow model. International Journal of Electrical and Computer Engineering (IJECE), 10(4), pp.4381-4389.

42. Aswini, N. and Uma, S.V., 2019. Obstacle avoidance and distance measurement for unmanned aerial vehicles using monocular vision. International Journal of Electrical and Computer Engineering, 9(5), p.3504.

43. Imran, A.Z.M., Hakim, M.L., Ahmed, M., Islam, M.T. and Hossain, E., 2021. Design of microstrip patch antenna to deploy unmanned aerial vehicle as UE in 5G wireless network. International Journal of Electrical \& Computer Engineering (2088-8708), 11(5).

44. Godse, S.P. and Mahalle, P.N., 2018. A Computational Analysis of ECC Based Novel Authentication Scheme in VANET. International Journal of Electrical \& Computer Engineering (2088-8708), 8(6).

45. Khamayseh, Y., Yassein, M.B. and Abu-Jazoh, M., 2019. Intelligent black hole detection in mobile AdHoc networks. International Journal of Electrical and Computer Engineering, 9(3), p.1968.

46. Cauvery, N.K., 2020. Trust-based secure routing against lethal behavior of nodes in wireless adhoc network. International Journal of Electrical \& Computer Engineering (2088-8708), 10(2).

47. Alamsyah, A., Purnama, I.K.E., Setijadi, E. and Purnomo, M.H., 2019. MPR selection to the OLSR quality of service in MANET using minmax algorithm. International Journal of Electrical and Computer Engineering, 9(1), p.417.

48. BK, C., Siddappa, M. and HS, J., 2020. Novel framework using dynamic passphrase towards secure and energy-efficient communication in Manet. International Journal of Electrical \& Computer Engineering (20888708), 10(2). 